\title{
Serum from hepatectomized rats induces the differentiation of adipose tissue mesenchymal stem cells into hepatocyte-like cells and upregulates the expression of hepatocyte growth factor and interleukin- 6 in vitro
}

\author{
JIANGYANG SUN $^{1 *}$, YUFENG YUAN $^{1 *}$, HAIQUAN QIN ${ }^{1}$, CHENGCHENG YING $^{2}$, \\ WEIJUN LIU ${ }^{1}$, JIN ZHANG ${ }^{1}$, YUEMING HE ${ }^{1}$ and ZHISU LIU ${ }^{1}$
}

Departments of ${ }^{1}$ General Surgery, ${ }^{2}$ Urology Surgery, Zhongnan Hospital of Wuhan University, Wuhan, Hubei 430071, P.R. China

Received October 27, 2012; Accepted December 19, 2012

DOI: 10.3892/ijmm.2013.1257

\begin{abstract}
Adipose tissue-derived mesenchymal stem cells (AT-MSCs) are an attractive alternative for clinical application due to their minimally invasive accessibility and availability in the body. However, the hepatic differentiation efficiency of AT-MSCs is insufficient for therapeutic application and the role of extrahepatic stem cells in liver regeneration remains poorly understood. This study was designed to investigate the effects of serum from rats subjected to $70 \%$ partial hepatectomy (PH) on the differentiation ability of rat AT-MSCs in vitro, and to explore the potential role of AT-MSCs in vivo following PH injury. Results showed that AT-MSCs treated with serum collected from rats $24 \mathrm{~h}$ after $70 \% \mathrm{PH}$ differentiated into hepatocyte-like cells, resembled hepatocyte-like cells with round or polygonal shape, expressed $\alpha$-fetoprotein, secreted albumin, synthesized urea and acquired cytochrome P450 type 3A4 enzyme activity, and upregulated the expression of interleukin (IL)- 6 and hepatocyte growth factor (HGF) transiently in vitro, although the hepatic differentiation efficiency was extremely low. AT-MSC transplantation after $70 \%$ PH ameliorated liver injury and promoted liver
\end{abstract}

Correspondence to: Dr Zhisu Liu, Department of General Surgery, Zhongnan Hospital of Wuhan University, Wuhan, Hubei 430071, P.R. China

E-mail: liuzhisu53@163.com

*Contributed equally

Abbreviations: $\mathrm{PH}$, partial hepatectomy; AFP, $\alpha$-fetoprotein; ALB, albumin; ALT, alanine aminotransferase; AST, aspartate aminotransferase; DMEM, Dulbecco's modified Eagle's medium; FBS, fetal bovine serum; DAPI, 4',6-diamidino-2-phenylindole; FITC, fluorescein isothiocyanate; GFP, green fluorescent protein; IL, interleukin; mRNA, messenger RNA; MSC, mesenchymal stem cell; PBS, phosphate-buffered saline; RT-PCR, reverse transcription polymerase chain reaction; CYP3A4, cytochrome P450 type 3A4

Key words: mesenchymal stem cells, interleukin 6, hepatocyte growth factor, hepatic differentiation, partial hepatectomy, transplantation regeneration, but did not increase the serum levels of IL-6 and HGF in vivo. This result suggests that the therapeutic effect of AT-MSCs in vivo after $24 \mathrm{~h}$ of $70 \% \mathrm{PH}$ does not increase IL-6 and HGF expression.

\section{Introduction}

Accumulating evidence suggests that adipose tissue-derived mesenchymal stem cells (AT-MSCs) possess the ability to transdifferentiate into other cell types including hepatocytes, similar to bone marrow-derived stem cells (BMSCs). Previous studies have shown that AT-MSCs differentiate into hepatocyte-like cells by culture with appropriate culture conditions, such as various cytokine mixtures (1-5). However, the efficacy of the hepatic differentiation of AT-MSCs is insufficient for therapeutic application and the role of extrahepatic stem cells in liver regeneration remains poorly understood. A successful differentiation is generally achieved by the step-by-step addition of growth factors, cytokines, and hormones, which emulate the sequence of events occurring during in vivo hepatogenesis (6). Therefore, studies have been conducted to explore novel means of facilitating differentiation of MSCs into hepatocytes. At present, no induction method can completely represent or mimic the pathological environment of the various types of liver diseases, as there is a marked regulation and complicated interaction in vivo under certain pathological environments. For example, liver regeneration after partial hepatectomy $(\mathrm{PH})$ injury is an extremely complex and well-orchestrated phenomenon (7). PH triggers a sequence of events that proceed in an orderly manner and are observed from the first $5 \mathrm{~min}$ to 5-7 days, and induces rapid induction of $>100$ genes not expressed in normal liver (8). A number of cytokines are upregulated during acute liver injury, including tumor necrosis factor- $\alpha$ (TNF- $\alpha$ ), interleukin-6 (IL-6), hepatocyte growth factor (HGF), transforming growth factor- $\alpha$ (TGF- $\alpha$ ), macrophage inflammatory protein-2 (MIP-2), stem cell factor (SCF), and various other cytokines (9-11).

Changes in the concentrations of the above signaling molecules in the plasma likely account for the fact that the signals for liver regeneration are transmitted by blood in pairs of parabiotic rats (12) or isolated hepatocytes in the 
adipose tissue when the orthotopic liver is subjected to partial hepatectomy (13). By mimicking the injured liver microenvironment, Wang et al (14) isolated BMSCs to induce differentiation of these cells into hepatocyte-like cells with HGF in vitro. Yang et al also reported that serum from liver radiofrequency ablation (RFA)-treated rats induced the differentiation of BMSCs into hepatic progenitor cells more efficiently compared with HGF (15), suggesting that serum under certain pathological environments may provide the ideal induction conditions to study unknown events that BMSCs may be involved in in vivo. Therefore, the present study aimed to determine whether serum from hepatectomized rats is able to induce the differentiation of AT-MSCs into hepatocyte-like cells, and to explore the possible role of AT-MSCs in vivo during liver regeneration.

\section{Materials and methods}

Materials. Low-glucose Dulbecco's modified Eagle's medium (L-DMEM), fetal bovine serum (FBS) (both were from Invitrogen Life Technologies, Carlsbad, CA, USA), and $0.25 \%$ trypsin-ethylenediaminetetraacetic acid (EDTA; Sino-American Company, Shanghai, China) were used for cell cultures. All other chemicals were purchased from SigmaAldrich (St. Louis, MO, USA), unless otherwise specified.

Experimental animals. Sixty-five male Sprague-Dawley (SD) rats weighing 140-160 g were obtained from the Laboratory Animals Center of Wuhan University (Wuhan, Hubei, China). Experiment procedures were carried out in accordance with the Code of Ethics of the World Medical Association.

Isolation and culture of AT-MSCs. AT-MSCs were isolated from the groin adipose tissue of SD rats. The capillaries of the adipose tissue were removed and washed three times with phosphate-buffered solution (PBS) under aseptic conditions. The adipose tissue was then minced into $3-5 \mathrm{~mm}$ sections using scissors and scalpels prior to digestion using $0.1 \%$ collagenase solution for $50 \mathrm{~min}$ in a water bath at $37^{\circ} \mathrm{C}$. The mixture was centrifuged at $1,500 \mathrm{rpm}$ for $8 \mathrm{~min}$ at room temperature $\left(25^{\circ} \mathrm{C}\right)$, and the supernatant containing adipose tissue was discarded. After the collected cells were washed three times with PBS (1,500 rpm, $8 \mathrm{~min})$, they were resuspended in L-DMEM containing $15 \%$ FBS, $1 \%$ L-glutamine, and $1 \%$ penicillin and streptomycin. Cells $\left(1 \times 10^{6}\right.$ cells $\left./ \mathrm{ml}\right)$ were seeded in culture flasks (T25; Corning Inc., Corning, NY, USA) and maintained in a humidified atmosphere of $95 \%$ air and $5 \% \mathrm{CO}_{2}$ at $37^{\circ} \mathrm{C}$. When the cells reached $80-90 \%$ confluence, they were collected with $0.25 \%$ Trypsin-EDTA and diluted $1: 2$ or 1:3 at each passage.

Flow cytometry (FACS) analysis. AT-MSCs of passage 3 (P3) were analyzed using a FACSCalibur (FC500) Flow Cytometer (Becton-Dickinson, San Jose, CA, USA). PE-conjugated CD34 (Santa Cruz Biotechnology, Inc., Santa Cruz, CA, USA), and fluorescein isothiocyanate (FITC)-conjugated CD44, CD45 and CD90 (AbD Serotec) antibodies were employed.

Differentiation potential of AT-MSCs. AT-MSCs from P3 to passage 5 (P5) were seeded into 6-well plates at $3 \times 10^{4}$ cells $/ \mathrm{cm}^{2}$ and grown to $90 \%$ confluence for the differentiation studies mentioned above. The cells were treated with each induction medium for at least two weeks, changing the medium twice a week.

Adipogenic differentiation protocol. The adipogenic medium consisted of L-DMEM supplemented with $10 \%$ FBS, $0.5 \mathrm{mM}$ 3-isobutyl-1-methylxanthine, $1 \mu \mathrm{M}$ dexamethasone, $200 \mu \mathrm{M}$ indomethacin, $10 \mu \mathrm{g} / \mathrm{ml}$ bovine insulin, and $1 \%$ penicillin and streptomycin.

Osteogenic differentiation protocol. Osteogenic medium consisted of L-DMEM supplemented with 10\% FBS, $0.1 \mathrm{M}$ dexamethasone, $0.3 \mathrm{mM}$ ascorbic acid, and $10 \mathrm{mM} \beta$-glycerol phosphate.

Hepatic differentiation protocol. Hepatic differentiation was induced over a period of two weeks based on the published differentiation protocols (16) with minor modifications. The hepatic differentiation medium consisted of L-DMEM supplemented with $10 \% \mathrm{FBS}, 10 \mu \mathrm{g} / \mathrm{ml}$ transferrin, $10 \mu \mathrm{g} / \mathrm{ml}$ insulin, $0.1 \mathrm{mM}$ ascorbic acid, $0.1 \mu \mathrm{M}$ dexamethasone, $10 \mathrm{ng} / \mathrm{ml}$ HGF (PeproTech; Rocky Hill, NJ, USA), $10 \mathrm{ng} / \mathrm{ml}$ bFGF (PeproTech), and $10 \mathrm{ng} / \mathrm{ml}$ OSM (PeproTech).

Immunofluorescence analysis. On days 7 and 14, samples of cultured cells were rinsed and fixed with $4 \%$ paraformaldehyde at $4^{\circ} \mathrm{C}$ for $30 \mathrm{~min}$. The cells were permeabilized with $0.1 \%$ Triton X-100 for $15 \mathrm{~min}$. After blocking the fixed cells for $30 \mathrm{~min}$ at $37^{\circ} \mathrm{C}$ with $1 \%$ bovine serum albumin (ALB), the cells were incubated overnight at $4^{\circ} \mathrm{C}$ in a humidity chamber with the respective primary antibodies: rabbit polyclonal antiALB (1:100) and goat polyclonal anti- $\alpha$-fetoprotein (AFP; 1:100) (both from Santa Cruz Biotechnology, Inc.). The cells were then incubated with the corresponding secondary antibody: rhodamine (TRITC)-conjugated donkey anti-rabbit IgG (1:100) and FITC-conjugated Affinipure donkey anti-goat IgG (1:100) (both from Proteintech Group, Inc., Chicago, IL, USA) for $30 \mathrm{~min}$ at room temperature. After rinsing, the nuclei were counterstained with $5 \mu \mathrm{g} / \mathrm{ml}$ 4',6-diamidino-2-phenylindole (DAPI; Beyotime, China). The cells were then visualized with a fluorescence microscope (Olympus BX51, Japan), and the images were analyzed using Image-Pro Plus 6.0.

Hepatic basal function evaluation of the differentiation cells. Cells differentiated on days $0,3,6,9,12,15,18,21$ and 24 were extensively washed with PBS, and then incubated with L-DMEM containing $5 \mathrm{mM} \mathrm{NH}_{4} \mathrm{Cl}$ overnight (12 h) in $5 \% \mathrm{CO}_{2}$ at $37^{\circ} \mathrm{C}$ prior to analysis. The supernatant was collected and the urea concentration was measured using a colorimetric assay kit (BioVision, USA) according to the manufacturer's instructions.

ALB production was evaluated on days $0,6,12,18$ and 24 using a quantitative enzyme-linked immunoassay kit (rat albumin ELISA; ALPCO Diagnostics, Salem, NH, USA) according to the manufacturer's instructions.

Cytochrome P450 type 3A4 (CYP3A4) enzyme activity was assessed by measurement of luciferase activity with the P450-Glo ${ }^{\mathrm{TM}}$ CYP3A4 Assay (Luciferin-PFBE; Promega, Madison, WI, USA) according to the manufacturer's instructions. Briefly, the cells were cultured in L-DMEM supplemented with $1 \mathrm{mmol} / 1$ phenobarbital (Sigma) for $48 \mathrm{~h}$ with a daily medium change. The cells were incubated at 
Table I. Primer sequences and product sizes.

\begin{tabular}{|c|c|c|c|c|}
\hline Gene name & & Primer sequences & Product size (bp) & $\mathrm{T}\left({ }^{\circ} \mathrm{C}\right)$ annealing \\
\hline IL-6 & $\begin{array}{l}\text { Sense } \\
\text { Antisense }\end{array}$ & $\begin{array}{l}\text { 5'-GCCTTCTTGGGACTGATGT-3' } \\
\text { 5'-TGAGTTGGATGGTCTTGGT-3' }\end{array}$ & 541 & 61 \\
\hline $\mathrm{HGF}$ & $\begin{array}{l}\text { Sense } \\
\text { Antisense }\end{array}$ & $\begin{array}{l}\text { 5'-ACATCACTCCCGAGAACTT-3' } \\
\text { 5'-AAACTAACCATCCACCCTAC-3' }\end{array}$ & 520 & 61 \\
\hline GAPDH & $\begin{array}{l}\text { Sense } \\
\text { Antisense }\end{array}$ & $\begin{array}{l}\text { 5'-CAGTGCCAGCCTCGTCTCAT-3' } \\
\text { 5'-AGGGGCCATCCACAGTCTTC-3' }\end{array}$ & 595 & 65 \\
\hline
\end{tabular}

IL-6, interleukin-6; HGF, hepatocyte growth factor; GAPDH, glyceraldehyde 3-phosphate dehydrogenase.

$37^{\circ} \mathrm{C}$ in L-DMEM supplemented with $50 \mu \mathrm{mol} / 1$ luciferin PFBE (150 $\mu \mathrm{l} /$ well). After $3 \mathrm{~h}$ of incubation, $50 \mu \mathrm{l}$ of medium was transferred in a opaque white 96-well plate and mixed with $50 \mu \mathrm{l}$ of luciferin detection reagent to initiate luminescent reaction. After 20-min incubation at room temperature, luminescence was measured with a Victor 3 luminometer (Perkin-Elmer, Boston, MA, USA).

Establishment of $70 \% \mathrm{PH}$ and collection of post-hepatectomy serum. The $70 \% \mathrm{PH}$ procedure was performed through a midline abdominal incision (17) under anesthesia with sodium pentobarbital $(35-40 \mathrm{mg} / \mathrm{kg}$, i.m. $\mathrm{n}=5)$. For the sham operation $(n=5)$, a similar surgical approach was used but only a portion of the xiphoid was excised. Approximately $24 \mathrm{~h}$ after surgery, blood samples were collected from the ventricle. The blood samples were placed at $4^{\circ} \mathrm{C}$ for $2 \mathrm{~h}$ and centrifuged at $3,000 \mathrm{rpm}$ for $10 \mathrm{~min}\left(4^{\circ} \mathrm{C}\right)$. The upper layer of each blood sample was aliquoted into microcentrifuge tubes and stored at $-80^{\circ} \mathrm{C}$ for future use. Surgery was performed using sterile surgical techniques. Surgical procedures were performed between 8 and 11 a.m. to minimize the influence of diurnal rhythms on subsequent hepatocyte proliferation.

AT-MSCs treated with post-hepatectomy serum. P3-P5 AT-MSCs were induced to differentiate under the following conditions: i) FBS group, L-DMEM supplemented with $10 \%$ FBS; ii) serum group, L-DMEM supplemented with $70 \%$ $\mathrm{PH}$ serum to a final concentration of $10 \%$; iii) HGF group, induction composition is the same as the hepatic differentiation medium; iv) Sham group, L-DMEM supplemented with serum from rats that had undergone the sham operation to a final concentration of $10 \%$. On days 7 and 14, the cell type was identified. The abovementioned induction experiments were repeated using three sets of serum (the serum collected from one rat representing one set).

AT-MSC transplantation following $P H$. Approximately $24 \mathrm{~h}$ after $70 \% \mathrm{PH}, \sim 1 \times 10^{6}$ cells/rat in $200 \mu \mathrm{l}$ of PBS were transfused into the tail vein with a 30 -gauge needle for $>1$ min (AT-MSC group, $n=12$ ). Rats treated with PBS alone (PBS group, $n=12$ ) and untreated rats (untreated group, $n=12$ ) served as the control groups. The animals were sacrificed at 48 and $72 \mathrm{~h}$ after $\mathrm{PH}$ ( $\mathrm{n}=6$ for each time-point in all treatment groups). Blood samples were collected from the ventricle and the residual livers were harvested and weighed, and then properly preserved for the subsequent procedures.

IL-6 and HGF concentration analysis by ELISA. On days 3 and 6 of the cell culture, the culture medium was removed and the cells were washed twice with PBS and replenished with serum-free L-DMEM. After $24 \mathrm{~h}$, the culture supernatant was harvested and centrifuged at 3,000 rpm for $10 \mathrm{~min}$ at $25^{\circ} \mathrm{C}$. The concentrations of IL- 6 and HGF were measured following the manufacturer's instructions using commercial ELISA kits (Quantikine Mouse IL-6 ELISA kit and Mouse HGF Duoset ELISA Development kit; Biotechnology Systems). The cells in the plate were harvested by $0.25 \%$ trypsin-EDTA digestion, and the number of cells was determined by hematocytometer counts. The total RNA was then isolated.

Reverse transcription polymerase chain reaction (RT-PCR) analysis. Total RNA was isolated from AT-MSCs cultured in each group for mRNA expression analysis. cDNA synthesis and the polymerase chain reaction (PCR) were performed using the ReverTra Ace Reverse Transcriptase kit (Toyobo Co., Ltd., Tokyo, Japan) and the Hot Start PCR kit (Takara), respectively, following the manufacturer's instructions. The PCR primer sequences and conditions are shown in Table I. The PCR reaction was 30 cycles.

Assessment of liver functions. The serum alanine aminotransferase (ALT), aspartate aminotransferase (AST), and ALB, were measured using a multiple serum biochemical analyzer (Hitachi, Japan).

Liver regeneration rate. The total body weight of each rat that fasted was weighed before it was sacrificed. The residual liver was then excised and weighed. The ratio of the residual liver weight to the whole body weight was utilized instead of the weight of the residual liver alone.

Histology and immunohistochemistry. For conventional morphological evaluation, liver tissue was fixed with $10 \%$ formalin, embedded with paraffin, sectioned, and stained with hematoxylin and eosin using standard histological techniques. The sections were also immunostained for Ki-67 according to the manufacturer's instructions. The samples were coun- 

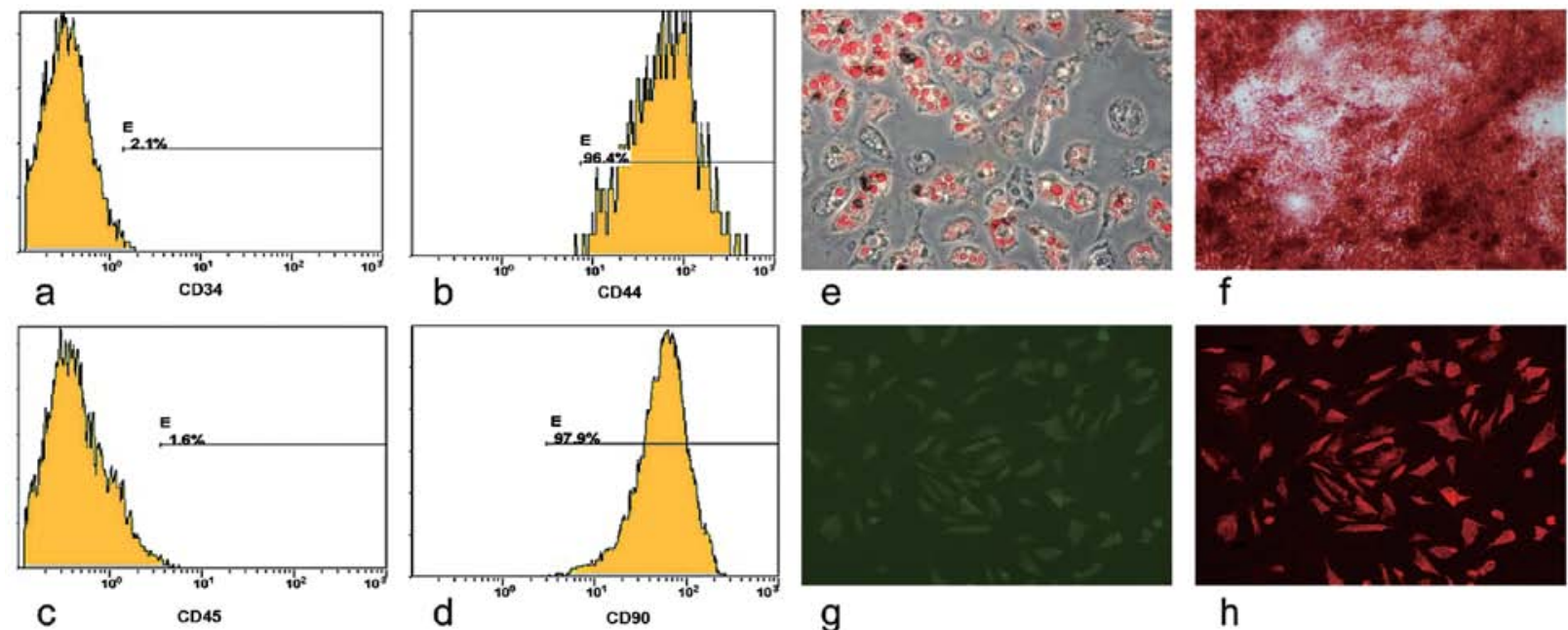

h

Figure 1. Cell surface markers and multi-lineage differentiation of isolated AT-MSCs. AT-MSCs were positive for (b) CD44 and (d) CD90 and negative for (a) CD34 and (c) CD45. (e) Adipogenic differentiation (Oil Red O staining, x200). (f) Osteogenic differentiation (Alizarin-Red staining, x200). (g and h) Hepatic differentiation, AFP (green) and ALB (red). Immunofluorescent staining shows the cells on the slide (x400).
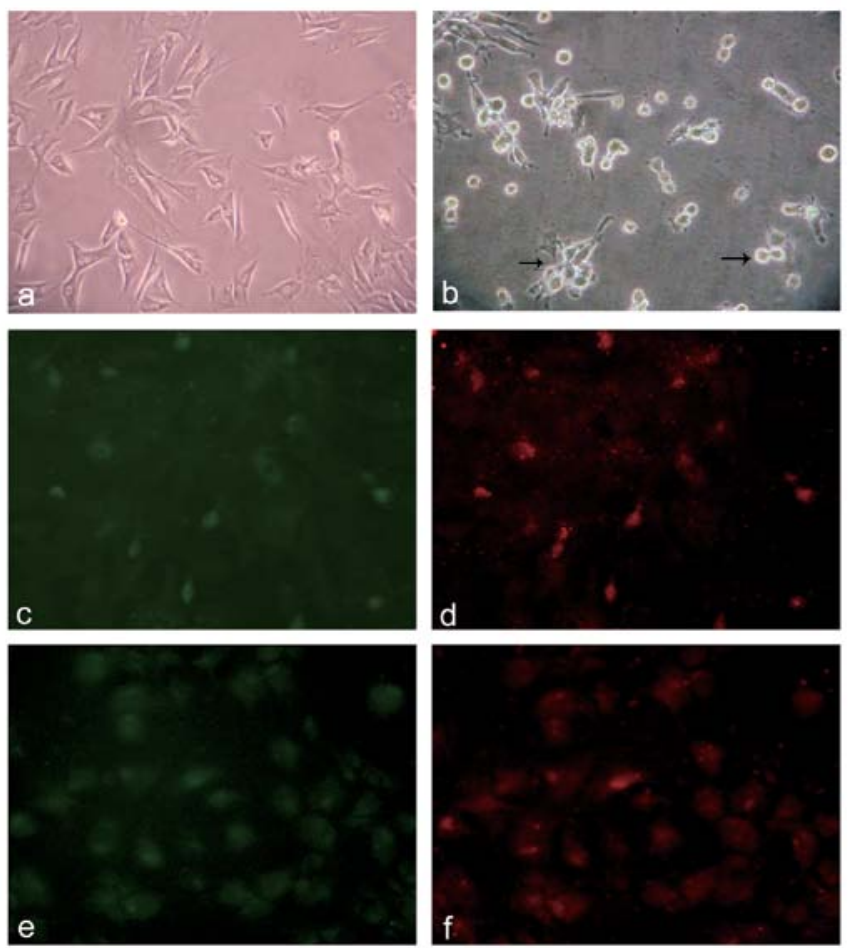

Figure 2. AT-MSCs were treated for two weeks with post-hepatectomy serum. Two weeks later, some of the spindle-shaped AT-MSCs gradually transformed into hepatocyte-like round or polygonal cells, the ratio of nucleoplasm decreased, adhesion ability weakened and was associated with a reduced number of cells in the serum group at the time of medium change. (a) Prior to induction, (b) two weeks after induction in the serum group. (e and f) More positive cells were observed in the HGF group compared with (c and d) the serum group. (Positive cell ratio was $53.12 \pm 0.76$ vs. $24.35 \pm 2.0, \mathrm{P}<0.001$ ). The positive cells were counted in at least five randomly selected high-power fields $(x 400)$ per slide.

terstained with hematoxylin. The Ki-67-positive cells were counted in at least five randomly selected high-power fields (x400) per slide.
Statistical analysis. Data are presented as the mean \pm standard deviations. Statistical significance was calculated using the SPSS 19.0 program. The Student's t-test was used for the pairwise comparisons and one-way ANOVA was used for the multiple comparisons. $\mathrm{P}<0.05$ was considered statistically significant.

\section{Results}

Characterization of AT-MSCs. The FACS analysis demonstrated that AT-MSCs expressed CD44 and CD90 (Fig. 1b and d), but not CD34 and CD45 (Fig. 1a and c). Two weeks after their exposure to adipogenic and four weeks after exposure to osteogenic induction medium, intracellular lipid droplets (Fig. 1e) and extracellular calcium phosphate precipitates (Fig. 1f) were observed using Oil Red O and Alizarin-Red staining. Two weeks after the induction of hepatic differentiation, AFP (green) and ALB (red) were observed using immunofluorescence (Fig. $1 \mathrm{~g}$ and $\mathrm{h}$ ).

Morphological observations and immunofluorescence staining of AT-MSCs cultured with post-hepatectomy serum. After culturing the cells with the serum for one week, some spindle-shaped cells transformed into round or polygonal hepatocyte-like cells gradually, the ratio of the nucleoplasm decreased, and adhesion ability weakened and was associated with a reduced number of cells in the serum group at the time of the medium change (Fig. $2 \mathrm{a}$ and b). By contrast, the cells in the FBS and Sham groups maintained the spindle-shaped phenotype of AT-MSCs. Following the two-week induction, the green fluorescence of the FITC-labeled AFP and the red fluorescence of the TRITC-labeled ALB were observed both in the serum and HGF groups (Fig. 2c-f). However, more positive cells were observed in the HGF group compared with the serum group (positive cell ratio, $53.12 \pm 0.76$ vs. $24.35 \pm 2.0, \mathrm{P}<0.001$ ).

ALB secretion, urea synthesis and CYP3A4 enzyme activity. The cells produced ALB during differentiation and the 

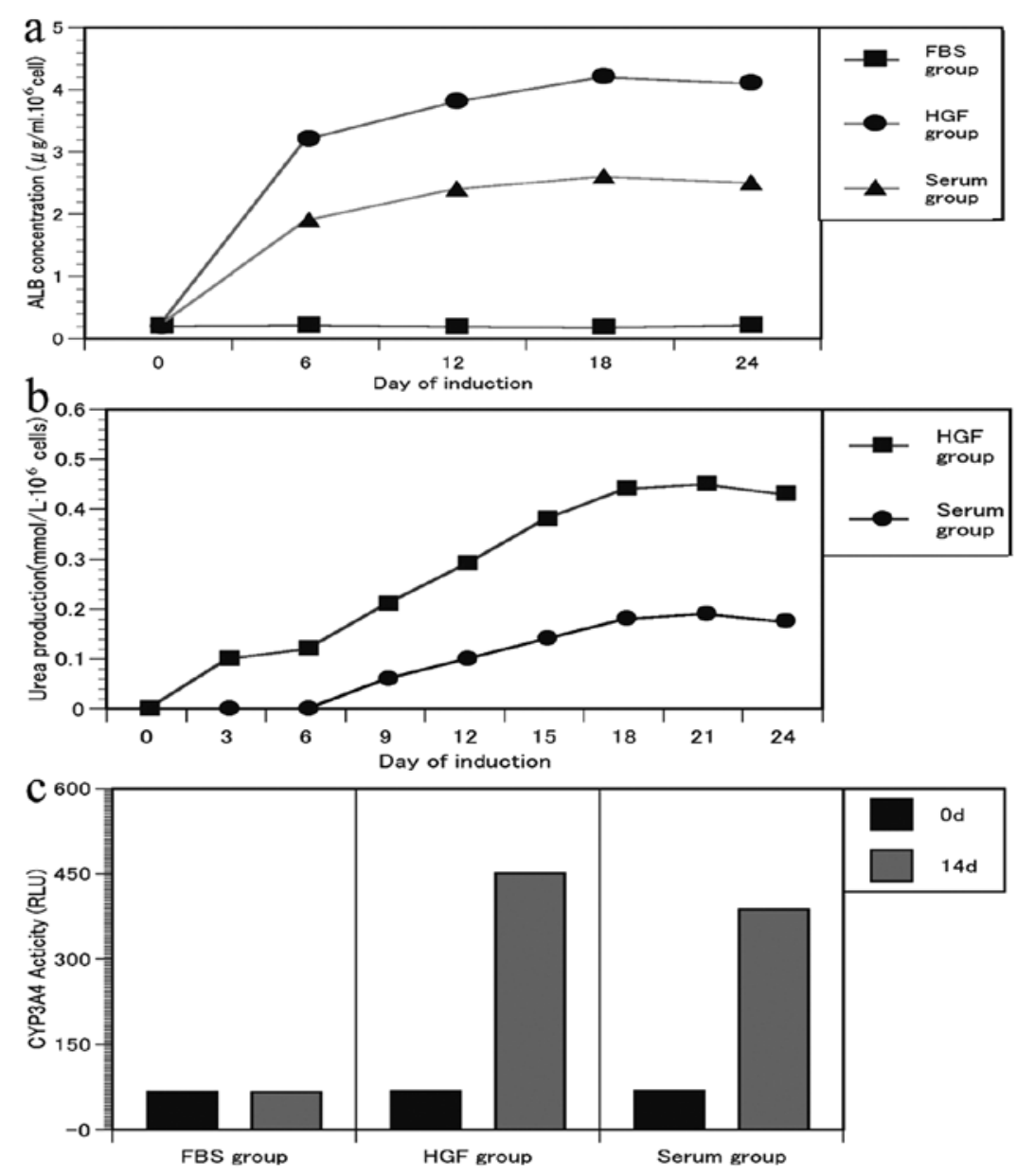

Figure 3. Functional characterization of AT-MSC-derived hepatocytes. Differentiation efficiency was evaluated at the functional level. (a) The cells in the HGF and serum groups produced ALB during differentiation and the secreted ALB increased 2-fold during the 24 days of differentiation. Urea levels gradually increased until day 21 in a time-dependent manner in the HGF and serum groups. (b) AT-MSCs in the FBS and Sham groups did not produce urea. (c) Activity of the CYP3A4 enzyme was significantly increased in differentiated AT-MSCs after 14 days of culture under differentiating conditions compared with undifferentiated AT-MSCs. The changes identified suggest that the AT-MSCs had undergone hepatocyte maturation.

secreted ALB increased 2-fold during the 24 days of differentiation (Fig. 3a). ALB was almost not detected in supernatants of the FBS group.

Urea production was present and the urea levels gradually increased until day 21 in a time-dependent manner in the HGF and serum groups (Fig. 3b). Cells in the FBS and Sham groups did not produce urea (data not shown).

Activity of the CYP3A4 enzyme was detected in differentiated AT-MSCs and was significantly enhanced when cells were cultured with $1 \mathrm{mmol} / 1$ phenobarbital compared with undifferentiated AT-MSCs (Fig. 3c). These changes suggest that the AT-MSCs had undergone hepatocyte maturation.

mRNA expression and cytokine concentration in culture supernatant. An increase in IL-6 and HGF levels was detected (day 3) in the culture supernatant in the HGF and serum groups (Fig. 4b) $(\mathrm{P}<0.01)$ compared with the FBS and Sham groups. However, these levels significantly decreased on day 6 $(\mathrm{P}<0.05)$, coinciding with the changes in mRNA expression (Fig. 4a).

Effect of AT-MSC transplantation on liver function after $70 \% \mathrm{PH}$. Approximately $24 \mathrm{~h}$ after AT-MSC transplantation, the ALT and AST levels decreased to a level lower than that of the PBS and untreated groups, and $\sim 48 \mathrm{~h}$ after the transplantation, this change became more pronounced (Fig. 5a and b) $(\mathrm{P}<0.001)$. Simultaneously, $48 \mathrm{~h}$ after AT-MSC transplantation, the ALB level in the AT-MSC group significantly increased compared with that in the PBS and untreated groups (Fig. 5c) $(\mathrm{P}<0.01)$. Thus, AT-MSC transplantation attenuated hepatocyte injury and improved the protein synthesis ability of the hepatocytes.

IL- 6 and HGF concentration in serum after AT-MSC transplantation. After $70 \% \mathrm{PH}$, the serum levels of HGF and IL-6 in the PBS and untreated groups peaked within $24 \mathrm{~h}$, gradually decreased, and then reached normal levels after $72 \mathrm{~h}$ (Fig. 5d and e). However, the HGF and IL-6 levels in the AT-MSC group were significantly decreased after $24 \mathrm{~h}$ of the transplantation $(\mathrm{P}<0.01)$ compared with the PBS and untreated groups. This decrease was even closer to that of their normal levels, showing that AT-MSC transplantation altered HGF and IL-6 expression in vivo.

Histological examination of livers. Approximately $24 \mathrm{~h}$ after $70 \% \mathrm{PH}$, the hepatic sinusoidal structure was well preserved in 

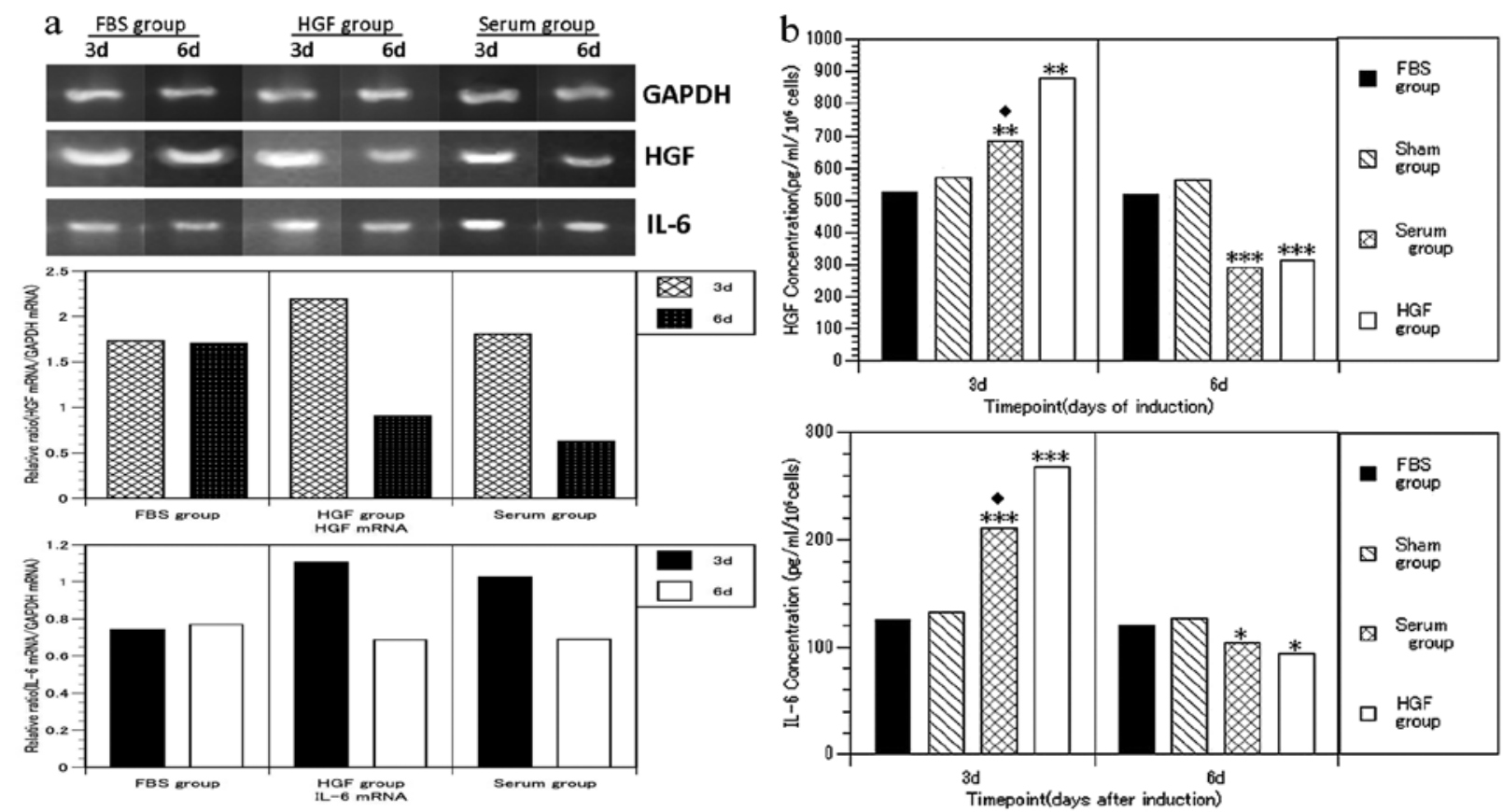

Figure 4. mRNA expression and HGF and IL-6 concentration in culture supernatant. (a) Total RNA of AT-MSCs was harvested 7 days (d7) and 14 days (d14) during induction and analyzed by RT-PCR for cytokine genes/growth factors. GAPDH was used as an internal reference to determine the expression of the FBS, HGF and PH groups. (b) HGF and IL-6 concentration change in the supernatant of culture cells. A transient increase (day 3) was noted in the culture supernatant in the HGF and serum groups. $\left({ }^{* * * *} \mathrm{P}<0.001,{ }^{* *} \mathrm{P}<0.01,{ }^{*} \mathrm{P}<0.05\right.$, compared with other groups, respectively; ${ }^{\bullet} \mathrm{P}<0.01$, serum group compared with HGF group).

all the samples, and inflammation or necrosis was not present. Histopathologic examination showed vacuolar degeneration (Fig. 6a, arrows) and mild central vein dilatation (Fig. 6a and c, asterisks). These features are commonly observed after liver resection. Approximately $24 \mathrm{~h}$ after AT-MSC transplantation, the injured livers were found to have less vacuolar degeneration than those in the PBS group (Fig. 6b), and the changes were more apparent $48 \mathrm{~h}$ after the transplantation (Fig. 6d). Approximately $48 \mathrm{~h}$ after $70 \% \mathrm{PH}$, all the groups exhibited a similar proliferation rate, although the proliferation rate $72 \mathrm{~h}$ after 70\% PH was significantly increased (Fig. 6e and f) in the AT-MSC group compared with the PBS group (Fig. 6g; Ki-67-positive ratio, $48.07 \pm 0.88$ vs. $35.33 \pm 0.81, \mathrm{P}<0.001$ ), and the residual liver weight was also increased in the AT-MSC group (Fig. 6h) $(\mathrm{P}<0.01)$.

\section{Discussion}

The 70\% PH model is a classical model for studying liver regeneration, in which many cytokines and growth factors that favor liver regeneration are released into the blood via the injured liver tissue. Different studies have suggested that various paracrine factors secreted by injured liver tissue have an important role in stem cell recruitment and engraftment. Those chemokines allow MSCs to enter the circulation prior to returning to the injured liver (18-20). In the present study, serum from $\mathrm{PH}$-induced acute liver injury was used as an agent to induce differentiation of AT-MSCs for the first time. As a result, these cells resembled hepatocyte-like cells with round or polygonal shape, expressed $\alpha$-fetoprotein, secreted ALB, synthesized urea, and acquired CYP450 3A4 enzyme activity, showing that these cells partially function as mature hepatocytes. However, the hepatic differentiation efficiency of AT-MSCs in the PH serum group was significantly lower than that in the HGF group. This result was different to that obtained by Yang et al (15) who showed that the serum collected after $24 \mathrm{~h}$ of $70 \% \mathrm{PH}$ did not exhibit appropriate culture conditions to promote the hepatic differentiation of AT-MSCs. It is known that in a typical wound healing scenario, the injury to the tissue results in disruption of capillary vascular networks and extravasation of blood, accompanied by local release of coagulation factors, platelets and growth factors (21). This does not occur after 70\% $\mathrm{PH}$. Various changes in hepatic blood flow patterns and oxygen partial pressure occur following the surgical removal of three liver lobes without damage to the two residual lobes. The two types of pathological processes of liver injury (PH and RFA injury) render the composition in the serum markedly different, leading to significantly different induction results, although the detailed mechanism of action remains to be elucidated.

The therapeutic effect of MSC transplantation is promising. However, studies have demonstrated that the rate of MSCs differentiating into liver cells in vivo was low $(22,23)$. Previous studies have suggested that $<3 \%$ of transplanted MSCs are attributed to the total organ mass (24-26). Moreover, even induced hepatocyte-like cells expressing a certain number of markers do not necessarily indicate that these cells possess similar functions to liver cells. MSCs act as 'trophic mediators' (27-32), which by secretion of bioactive factors act as either immunosuppressors or promoters of regeneration $(29,33,34)$. A previous study has also shown that AT-MSCs produce significantly more HGF, granulocyte colony-stimulating factor (G-CSF), granulocyte-macrophage colony-stimulating factor 

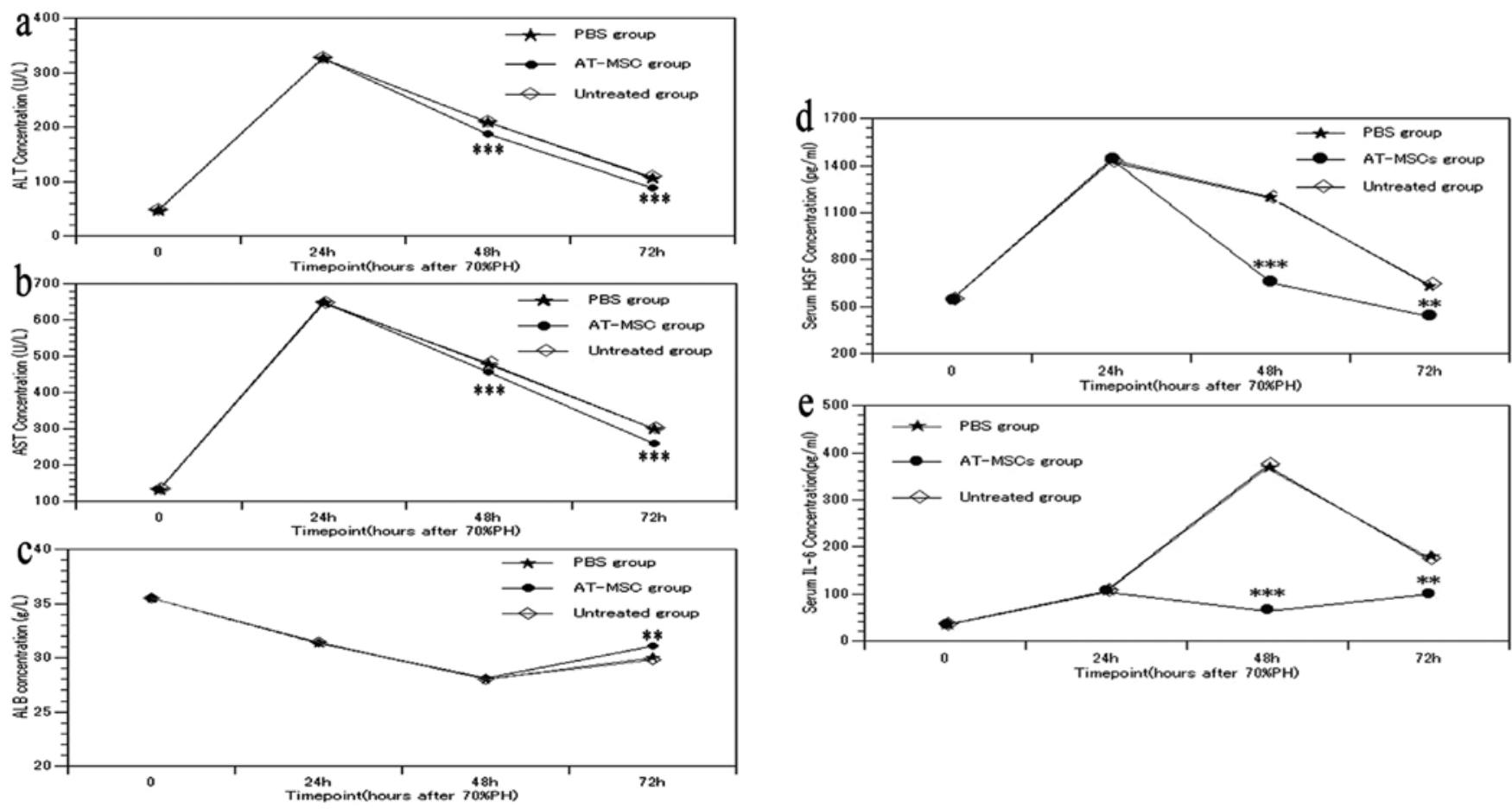

Figure 5. Liver function test and concentration of cytokines in post-hepatectomy serum at different time-points after 70\% PH. The levels of (a) ALT and (b) AST were slightly decreased in the AT-MSC group at $48 \mathrm{~h}$ after 70\% PH. (c) ALB levels were markedly increased in the AT-MSC group at $72 \mathrm{~h}$ after $70 \%$ PH. ${ }^{* * *} \mathrm{P}<0.001,{ }^{* *} \mathrm{P}<0.01$, compared with the PBS and untreated groups, respectively). (d) HGF and (e) IL-6 levels in the AT-MSC group were significantly decreased $24 \mathrm{~h}$ after the transplantation. ${ }^{* * *} \mathrm{P}<0.001,{ }^{* *} \mathrm{P}<0.01,{ }^{*} \mathrm{P}<0.05$, compared with the PBS and untreated group, respectively).
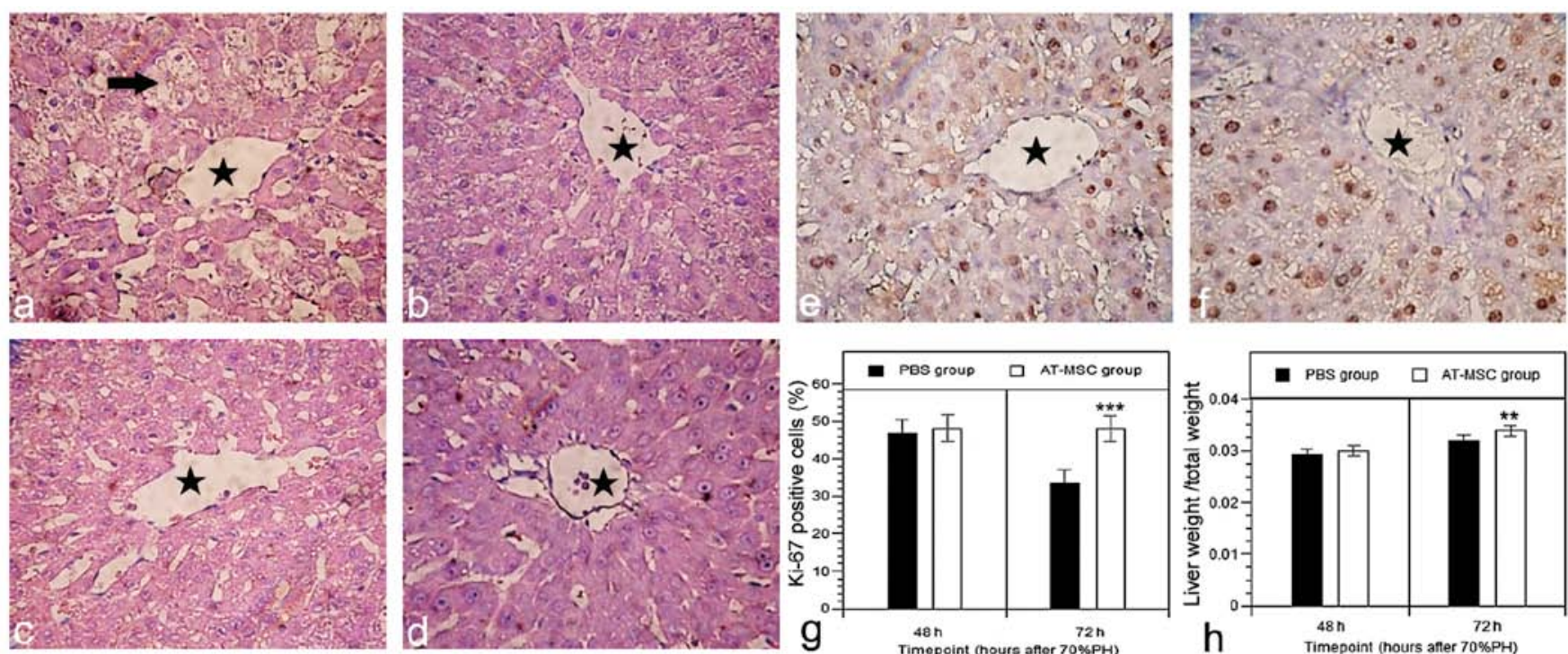

Figure 6. Histological examination at different time-points following AT-MSC administration with (a and d) hematoxylin and eosin staining (x400). The arrow shows vacuolar degeneration of hepatocytes, the star shows the central vein. (c) Twenty-four hours after AT-MSCs transplantation, (d) the injured livers were found to have less vacuolar degeneration, and the changes were more apparent $48 \mathrm{~h}$ after the transplantation compared with those in the PBS group. (a and b) Twenty-four and $48 \mathrm{~h}$ after PBS administration. (e and f) Immunohistochemistry staining of Ki-67 at $48 \mathrm{~h}$ after AT-MSCs administration (x400). The number of (g) Ki-67-positive cells and ratio of $(\mathrm{h})$ residual liver weight to the whole body weight $\left({ }^{* * *} \mathrm{P}<0.001,{ }^{* * *} \mathrm{P}<0.01\right.$ compared with the PBS group, respectively.).

(GM-CSF), and IL-6 compared with BMSCs and normal human dermal fibroblasts (NHDFs) (35). HGF is an important factor in the process of differentiation from BMSCs to hepatocytes and osteoblasts $(36,37)$, IL-6 is necessary and sufficient for enhanced MSC proliferation, and inhibits adipogenic and chondrogenic differentiation (38). Moreover, an increase in the mRNA expression levels of IL-6 was observed when BMSCs were co-cultured with hepatocytes from GaIN-induced injured liver (39). Therefore, we examined the expression of HGF and IL-6 during the induction process. 
In this study, we found that AT-MSCs briefly upregulated the mRNA expression of HGF and IL-6 when cultured with the post-hepatectomy serum. The concentration of HGF and IL-6 in the supernatant, as detected by ELISA, also conformed with the change of the mRNA expression. Previous studies have shown that HGF is a potent mitogen in vivo, and that an increasing HGF expression benefits liver injury attenuation $(40,41)$. It has also been reported that implantation of the human HGF-expressing MSCs, improves liver regeneration, reduces mortality in rats, and has a potent anti-fibrotic effect on the small-for-size liver transplantation model (42). Thus, the upregulated expression of HGF and IL- 6 may be the effector molecules involved in the promotion of liver regeneration in vivo. To validate our hypothesis, we transplanted AT-MSCs into the rat tail vein at $24 \mathrm{~h}$ after $70 \% \mathrm{PH}$ to observe the concentration changes of HGF and IL-6 in vivo. We found that AT-MSC transplantation significantly improved liver function and facilitated liver regeneration, but did not increase the serum levels of HGF and IL-6 as expected. By contrast, these results showed that a reduced concentration of HGF and IL-6 may be more beneficial for liver regeneration and function recovery, which is a notewothy finding to explain the reduction in HGF and IL-6 concentrations following AT-MSC transplantation.

However, the reason for the concentration of HGF and IL- 6 transiently increasing from 24 to $48 \mathrm{~h}$ after the transplantation remains to be verified, as well as whether other cytokines/growth factors are involved in the regeneration process. To clarify the aforementioned issues, more detailed studies are required. Recently, a new study (43) has demonstrated that the acutely transplanted neural stem/progenitor cells (NSPCs) had beneficial effects on spinal cord injury, particularly neuroprotection and neurohumoral secretion, whereas their in situ secretory activity differed significantly from that predicted in vitro. Authors of that study found that in the pathological environment, overall transcriptional activity, external signal transduction and neural differentiation of engrafted NSPCs were significantly suppressed. Those results emphasize the vulnerability of engrafted stem cells to environmental force, while emphasizing the importance of in situ analysis in advancing the efficacy and safety of stem cell-based therapies (43).

In conclusion, we have shown that serum from hepatectomized rats after $24 \mathrm{~h}$ of surgery triggered the differentiation of AT-MSCs into hepatocyte-like cells, although the hepatic differentiation efficacy was extremely low. AT-MSCs upregulated the expression of HGF and IL- 6 when cultured with the post-hepatectomy serum. However, they did not increase the serum levels of HGF and IL-6 when transplanted in vivo as we expected. This finding should therefore be studied further. However, there were limitations to our study. One was that we did not observe whether AT-MSCs are able to migrate into the injury liver and differentiate into hepatocytes. Another one was the concentration of the growth factors and cytokines in the blood markedly altered during the liver regeneration process, thus, serum from hepatectomized rats after $24 \mathrm{~h}$ of their operation did not exhibit the entire change in blood after $70 \%$ PH. Thus, AT-MSCs may act through multiple mechanisms to coordinate a marked integrated response to liver regeneration after $70 \% \mathrm{PH}$ in vivo. Therefore, the processes by which these cells act to decrease the serum levels of HGF and IL-6, as well as the mechanisms by which they act to reverse hepatocyte damage and promote liver regeneration in vivo remains to be investigated.

\section{Acknowledgements}

This study was supported by the Fundamental Research Funds for the Central Universities.

\section{References}

1. Banas A, Teratani T, Yamamoto Y, et al: Rapid hepatic fate specification of adipose-derived stem cells and their therapeutic potential for liver failure. J Gastroenterol Hepatol 24: 70-77, 2009.

2. Banas A, Teratani T, Yamamoto Y, et al: Adipose tissue-derived mesenchymal stem cells as a source of human hepatocytes. Hepatology 46: 219-228, 2007.

3. Sgodda M, Aurich H, Kleist S, et al: Hepatocyte differentiation of mesenchymal stem cells from rat peritoneal adipose tissue in vitro and in vivo. Exp Cell Res 313: 2875-2886, 2007.

4. Yamamoto Y, Banas A, Murata S, et al: A comparative analysis of the transcriptome and signal pathways in hepatic differentiation of human adipose mesenchymal stem cells. FEBS J 275: 1260-1273, 2008.

5. Talens-Visconti R, Bonora A, Jover R, et al: Hepatogenic differentiation of human mesenchymal stem cells from adipose tissue in comparison with bone marrow mesenchymal stem cells. World J Gastroenterol 12: 5834-5845, 2006.

6. Snykers S, Vanhaecke T, Papeleu P, et al: Sequential exposure to cytokines reflecting embryogenesis: the key for in vitro differentiation of adult bone marrow stem cells into functional hepatocyte-like cells. Toxicol Sci 94: 330-341, 2006.

7. Michalopoulos GK: Liver regeneration. J Cell Physiol 213: 286-300, 2007

8. Taub R: Liver regeneration: from myth to mechanism. Nat Rev Mol Cell Biol 5: 836-847, 2004.

9. Brand S, Beigel F, Olszak T, et al: IL-22 is increased in active Crohn's disease and promotes proinflammatory gene expression and intestinal epithelial cell migration. Am J Physiol Gastrointest Liver Physiol 290: G827-G838, 2006.

10. Cressman DE, Greenbaum LE, DeAngelis RA, et al: Liver failure and defective hepatocyte regeneration in interleukin-6-deficient mice. Science 274: 1379-1383, 1996.

11. Ren X, Hogaboam C, Carpenter A and Colletti L: Stem cell factor restores hepatocyte proliferation in IL-6 knockout mice following 70\% hepatectomy. J Clin Invest 112: 1407-1418, 2003.

12. Moolten FL and Bucher NL: Regeneration of rat liver: transfer of humoral agent by cross circulation. Science 158: 272-274, 1967.

13. Jirtle RL and Michalopoulos G: Effects of partial hepatectomy on transplanted hepatocytes. Cancer Res 42: 3000-3004, 1982.

14. Wang PP, Wang JH, Yan ZP, et al: Expression of hepatocyte-like phenotypes in bone marrow stromal cells after HGF induction. Biochem Biophys Res Commun 320: 712-716, 2004.

15. Yang Y, Qu B, Huo JH, Wu SL, Zhang MY and Wang ZR: Serum from radiofrequency-injured livers induces differentiation of bone marrow stem cells into hepatocyte-like cells. J Surg Res 155: 18-24, 2009.

16. Seo MJ, Suh SY, Bae YC and Jung JS: Differentiation of human adipose stromal cells into hepatic lineage in vitro and in vivo. Biochem Biophys Res Commun 328: 258-264, 2005.

17. Greene AK and Puder M: Partial hepatectomy in the mouse: technique and perioperative management. J Invest Surg 16: 99-102, 2003

18. Hatch HM, Zheng D, Jorgensen ML and Petersen BE: SDF-1alpha/CXCR4: a mechanism for hepatic oval cell activation and bone marrow stem cell recruitment to the injured liver of rats. Cloning Stem Cells 4: 339-351, 2002.

19. Dalakas E, Newsome PN, Harrison DJ and Plevris JN: Hematopoietic stem cell trafficking in liver injury. FASEB J 19: 1225-1231, 2005.

20. Gomez-Aristizabal A, Keating A and Davies JE: Mesenchymal stromal cells as supportive cells for hepatocytes. Mol Ther 17: 1504-1508, 2009. 
21. Schafer M and Werner S: Transcriptional control of wound repair. Annu Rev Cell Dev Biol 23: 69-92, 2007.

22. Mitchell $\mathrm{C}$ and Fausto N: Bone marrow-derived hepatocytes: rare but promising. Am J Pathol 161: 349-350, 2002.

23. Fausto N: Liver regeneration and repair: hepatocytes, progenitor cells, and stem cells. Hepatology 39: 1477-1487, 2004.

24. Malhi H and Gupta S: Hepatocyte transplantation: new horizons and challenges. J Hepatobiliary Pancreat Surg 8: 40-50, 2001.

25. Menthena A, Deb N, Oertel M, et al: Bone marrow progenitors are not the source of expanding oval cells in injured liver. Stem Cells 22: 1049-1061, 2004

26. Wang J, Clark JB, Rhee GS, Fair JH, Reid LM and Gerber DA: Proliferation and hepatic differentiation of adult-derived progenitor cells. Cells Tissues Organs 173: 193-203, 2003.

27. Caplan AI and Dennis JE: Mesenchymal stem cells as trophic mediators. J Cell Biochem 98: 1076-1084, 2006.

28. Parekkadan B, van Poll D, Suganuma K, et al: Mesenchymal stem cell-derived molecules reverse fulminant hepatic failure. PLoS One 2: e941, 2007.

29. Parekkadan B, van Poll D, Megeed Z, et al: Immunomodulation of activated hepatic stellate cells by mesenchymal stem cells Biochem Biophys Res Commun 363: 247-252, 2007.

30. Sadat S, Gehmert S, Song YH, et al: The cardioprotective effect of mesenchymal stem cells is mediated by IGF-I and VEGF. Biochem Biophys Res Commun 363: 674-679, 2007.

31. Gnecchi M, He H, Noiseux N, et al: Evidence supporting paracrine hypothesis for Akt-modified mesenchymal stem cell-mediated cardiac protection and functional improvement. FASEB J 20: 661-669, 2006

32. Shito M, Balis UJ, Tompkins RG, Yarmush ML and Toner M: A fulminant hepatic failure model in the rat: involvement of interleukin-lbeta and tumor necrosis factor-alpha. Dig Dis Sci 46: 1700-1708, 2001.

33. Gnecchi M, Zhang Z, Ni A and Dzau VJ: Paracrine mechanisms in adult stem cell signaling and therapy. Circ Res 103: 1204-1219, 2008 .
34. van Poll D, Parekkadan B, Cho $\mathrm{CH}$, et al: Mesenchymal stem cell-derived molecules directly modulate hepatocellular death and regeneration in vitro and in vivo. Hepatology 47: 1634-1643, 2008.

35. Banas A, Teratani T, Yamamoto Y, et al: IFATS collection: in vivo therapeutic potential of human adipose tissue mesenchymal stem cells after transplantation into mice with liver injury. Stem Cells 26: 2705-2712, 2008.

36. Miyazaki M, Akiyama I, Sakaguchi M, et al: Improved conditions to induce hepatocytes from rat bone marrow cells in culture. Biochem Biophys Res Commun 298: 24-30, 2002.

37. Wen Q, Zhou L, Zhou C, Zhou M, Luo W and Ma L: Change in hepatocyte growth factor concentration promote mesenchymal stem cell-mediated osteogenic regeneration. J Cell Mol Med 16: 1260-1273, 2012

38. Pricola KL, Kuhn NZ, Haleem-Smith H, Song Y and Tuan RS: Interleukin-6 maintains bone marrow-derived mesenchymal stem cell stemness by an ERK1/2-dependent mechanism. J Cell Biochem 108: 577-588, 2009.

39. Lam SP, Luk JM, Man K, et al: Activation of interleukin6-induced glycoprotein 130/signal transducer and activator of transcription 3 pathway in mesenchymal stem cells enhances hepatic differentiation, proliferation, and liver regeneration. Liver Transpl 16: 1195-1206, 2010.

40. Shiota $G$ and Kawasaki H: Hepatocyte growth factor in transgenic mice. Int J Exp Pathol 79: 267-277, 1998.

41. Ueki T, Kaneda Y, Tsutsui H, et al: Hepatocyte growth factor gene therapy of liver cirrhosis in rats. Nat Med 5: 226-230, 1999.

42. Yu Y, Lu L, Qian X, et al: Antifibrotic effect of hepatocyte growth factor-expressing mesenchymal stem cells in small-for-size liver transplant rats. Stem Cells Dev 19: 903-914, 2010.

43. Kumamaru $\mathrm{H}$, Ohkawa $\mathrm{Y}$, Saiwai $\mathrm{H}$, et al: Direct isolation and RNA-seq reveal environment-dependent properties of engrafted neural stem/progenitor cells. Nat Commun 3: 1140, 2012. 